\title{
Improving the efficiency of reactive case detection for malaria elimination in southern Zambia: A cross- sectional study
}

\section{CURRENT STATUS: UNDER REVISION}

Malaria Journal $\triangle B M C$

Fiona R.P. Bhondoekhan

Johns Hopkins University Bloomberg School of Public Health

Kelly M. Searle

University of Minnesota

Harry Hamapumba

Macha Research Trust

Mukuma Lubinda

Macha Research Trust

Japhet Matoba

Macha Research Trust

Michael Musonda

Macha Research Trust

Ben Katowa

Macha Research Trust

Timothy M. Shields

Johns Hopkins University Bloomberg School of Public Health

Tamaki Kobayashi

Johns Hopkins University Bloomberg School of Public Health

Douglas Norris

Johns Hopkins University Bloomberg School of Public Health

Frank C Curriero

Johns Hopkins University Bloomberg School of Public Health

Jennifer C. Stevenson 
Macha Research Trust

Philip E. Thuma

Macha Research Trust

William J. Moss

wmoss1@jhu.eduCorresponding Author

\section{DOI:}

$10.21203 / \mathrm{rs} .2 .21595 / \mathrm{v} 1$

\section{SUBJECT AREAS}

Infectious Diseases

\section{KEYWORDS}

malaria, Zambia, elimination, screening, reactive case detection, environment 
Abstract

Background: Reactive case detection (RCD) seeks to enhance malaria surveillance and control by identifying and treating parasitemic individuals residing near index cases. In Zambia, this strategy starts with passive detection of symptomatic incident malaria cases at local health facilities or by community health workers, with subsequent home visits to screen-and-treat residents in the index case and neighboring (secondary) households within a 140-meter radius using rapid diagnostic tests (RDTs). However, a small circular radius may not be the most efficient strategy to identify parasitemic individuals in low-endemic areas with hotspots of malaria transmission. To evaluate if RCD efficiency could be improved by increasing the probability of identifying parasitemic residents while screening fewer houses, we incorporated environmental risk factors and a larger screening radius (250 meters) in a region of low malaria endemicity.

Methods: Between January 12, 2015 and July 26, 2017, 4,170 individuals (residing in 158 index and 531 secondary households) were enrolled and completed a baseline questionnaire in the catchment area of Macha Hospital in Choma District, Southern Province, Zambia. Plasmodium

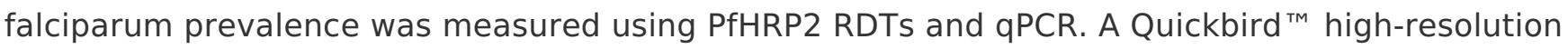
satellite image of the catchment area was used to create environmental risk factors in ArcGIS, and generalized estimating equations were used to evaluate associations between risk factors and secondary households with parasitemic individuals.

Results: The overall parasite prevalence in secondary (non-index case) households was $0.7 \%$ by RDT and $1.8 \%$ by qPCR. $8.5 \%$ (45) of secondary households had at least one resident with parasitemia by qPCR or RDT. The risk of a secondary household having a parasitemic resident was significantly increased in proximity to higher order streams and marginally with increasing distance from index households. The adjusted OR for proximity to third- and fifth-order streams were 2.97(95\% Cl: 1.04 $8.42)$ and $2.30(95 \% \mathrm{Cl}: 1.04-5.09)$, respectively, and that for distance to index households for each 50 meters was $1.24(95 \% \mathrm{Cl}: 0.98-1.58)$.

Conclusion: Applying proximity to streams as a screening tool, 16\% (3) more malaria-positive secondary households were identified than using a $140 \mathrm{~m}$ circular screening radius. This analysis 
highlights the potential use of environmental risk factors as a screening strategy to increase RCD efficiency.

Full Text

Due to technical limitations, full-text HTML conversion of this manuscript could not be completed. However, the manuscript can be downloaded and accessed as a PDF.

Figures 

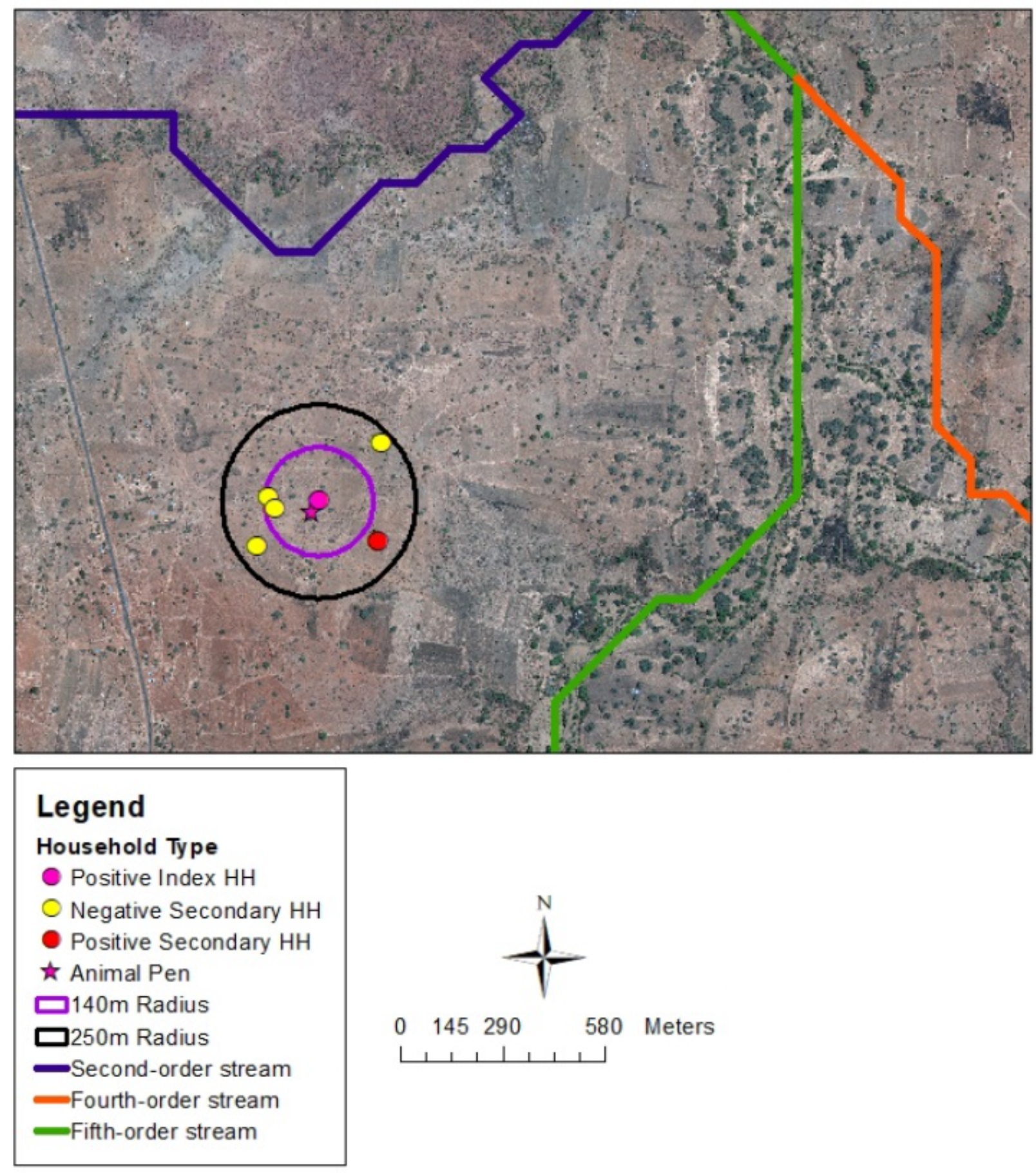

Figure 1

High-resolution Quickbird $^{\mathrm{TM}}$ satellite image for catchment area of Macha Hospital in Choma District. 


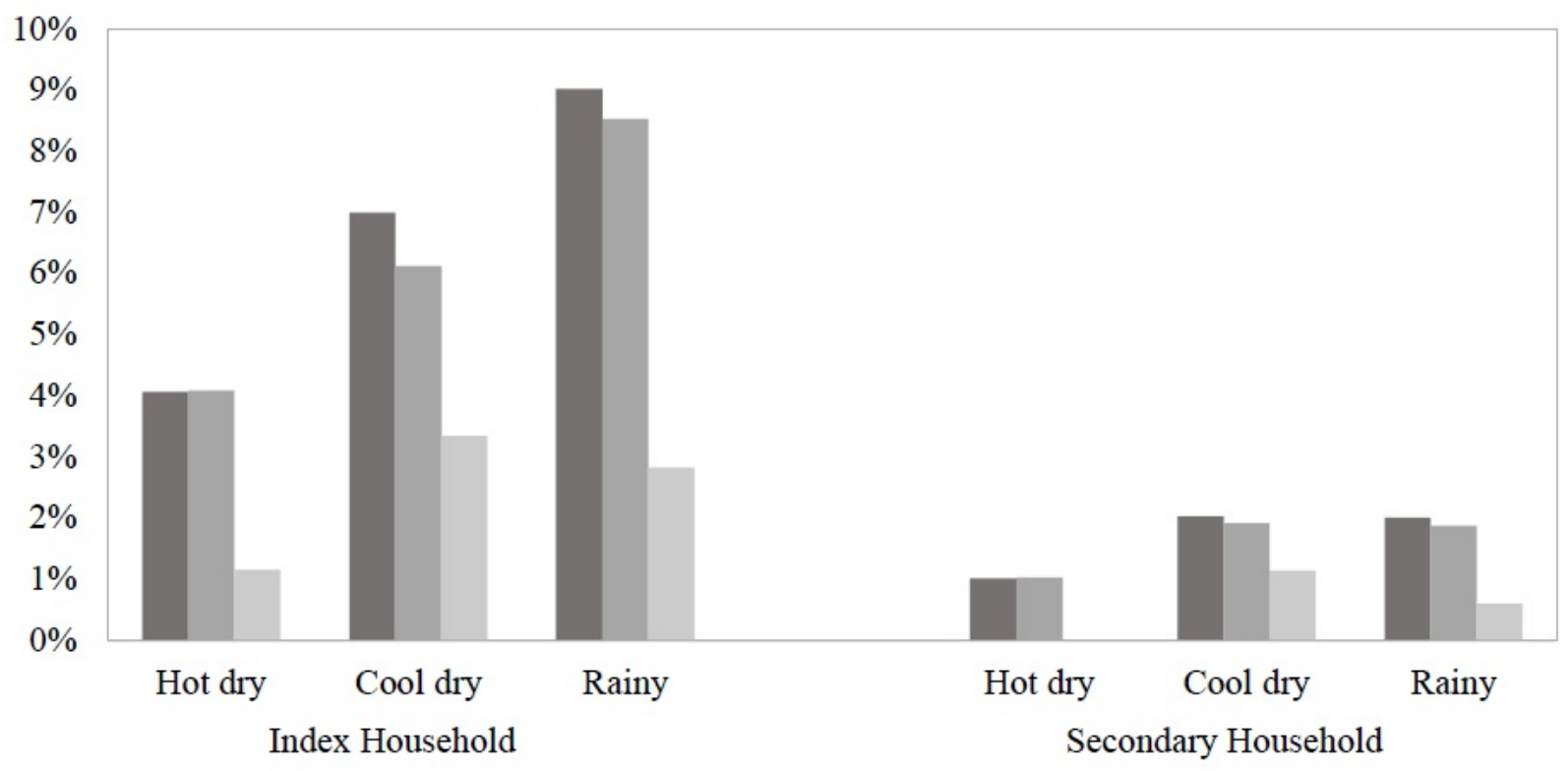

nOverall parasite prevalence $₫ \mathrm{qPCR}$ only parasite prevalence* $₫$ RDT only parasite prevalence** ${ }^{*} \mathrm{qPCR}=$ quantitative Polymerase Chain reaction; $* * \mathrm{RDT}=$ Rapid Diagnostic Test

Figure 2

Parasite prevalence (\%) for index and secondary households, January 2015 - July 2017 in southern Zambia. 